\title{
Overview of the FLUKA code
}

Giuseppe Battistoni ${ }^{\mathrm{a}}$, Till Boehlen ${ }^{\mathrm{c}}$, Francesco Ceruttic ${ }^{\mathrm{c}}$, Pik Wai Chin ${ }^{\mathrm{c}}$, Luigi Salvatore Esposito ${ }^{c}$, Alberto Fassò ${ }^{b}$, Alfredo Ferraric ${ }^{c}$, Anton Lechner ${ }^{c}$, Andrea Mairani ${ }^{\mathrm{e}}$, Alessio Mereghetti ${ }^{\mathrm{c}}$, Pablo Garcia Ortega ${ }^{\mathrm{c}}$, Johannes Ranft ${ }^{\mathrm{d}}$, Stefan Roesler ${ }^{\mathrm{c}}$, Paola R. Salac ${ }^{\mathrm{c}}$, Vasilis Vlachoudis ${ }^{\mathrm{c}, *}$

${ }^{a}$ INFN sezione di Milano, via Celoria 16, I-20133 Milano, Italy

${ }^{b}$ ELI Beamlines, Institute of Physics of the Academy of Sciences, Harfa Office Park, Ceskomoravska 2420/15a, 19093 Prague 9, Czech Republic ${ }^{c}$ CERN, CH-1211 Geneva 23, Switzerland

${ }^{d}$ Universität Gesamthochschule Siegen, Fachbereich Physik, D-57068 Siegen, Germany ${ }^{e}$ CNAO, Pavia, Italy

\section{Introduction}

The FLUKA code Battistoni et al. (2006b); Ferrari et al. (2005) is a general purpose Monte Carlo code for the interaction and transport of hadrons, heavy ions, and electromagnetic particles from few $\mathrm{keV}$ (or thermal energies for neutrons) to Cosmic Ray energies in arbitrary materials. It is built and maintained with the aim of including the best possible physical models in terms of completeness and precision. In this "microscopic" approach, each step has sound physical bases. Performances are optimized comparing with particle production data at single interaction level. No tuning whatsoever on "integral" data, like calorimeter resolutions, thick target yields etc., is performed. Therefore, final predictions are obtained with minimal free parameters, fixed for all energies and target/projectile combinations. Results in complex cases as well as scaling laws and properties come out naturally from the underlying physical models and the basic conservation laws are fulfilled "a priori". Moreover, the microscopic approach preserves correlations within interactions and among the shower components, and it provides predictions where no experimental data is directly available. When needed, powerful biasing techniques are available to reduce computing time.

Descriptions of FLUKA models and extensive benchmarking can be found in the literature (see the web page, http://www.fluka.org). FLUKA is jointly developed by the European Laboratory for Particle Physics (CERN) and the Italian Institute for Nuclear Physics (INFN).

*Corresponding author: Vasilis.Vlachoudis@cern.ch 


\section{Transport of electromagnetic particles and muons}

For historical reasons, FLUKA is best known for its hadron event generators, but it can handle with similar or better accuracy electromagnetic effects Fassò et al. (2001). The energy range covered by this sector of FLUKA is very wide: the program can transport photons and electrons over about 12 energy decades, from $1 \mathrm{PeV}$ down to $1 \mathrm{keV}$. Electromagnetic and hadronic cascades are fully coupled including low energy (i.e. $<20 \mathrm{MeV}$ ) neutron interactions.

The simulation of the electromagnetic cascade in FLUKA is very accurate, including the Landau-Pomeranchuk-Migdal effect and a special treatment of the tip of the bremsstrahlung spectrum. Electron pairs and bremsstrahlung are sampled from the proper double differential energy-angular distributions improving the common practice of using average angles. In a similar way, the three-dimensional shape of the electromagnetic cascades is reproduced in detail by a rigorous sampling of correlated energy and angles in decay, scattering, and multiple Coulomb scattering. Tabulations for pair production, photoelectric and total coherent cross-sections as well as for atomic form factor data are based on the EPDL97 Cullen et al. (1997) photon cross section library.

Bremsstrahlung, direct pair production and muon photonuclear interactions by muons are modeled according to state-of-the-art theoretical description and have been checked against experimental data.

\section{Charged particle transport}

Transport of charged particles is performed through an original Multiple Coulomb scattering algorithm Ferrari et al. (1992), supplemented by an optional single scattering method. The treatment of ionization energy loss is based on a statistical approach alternative to the standard Landau and Vavilov ones that provides a very good reproduction of average ionization and of fluctuations Fassò et al. (1997). An example of the ability of FLUKA in predicting energy loss spectra in thin detectors Bak et al. (1987) is presented in Figure 1.

Multiple scattering with inclusion of nuclear form factors is applied also to heavy ion transport. Up-to-date effective charge parameterizations are employed, and straggling of ion energy loss is described in "normal" first Born approximation with inclusion of charge exchange effects.

The precise determination of ion range and ionization losses is of utmost importance in dosimetry and in therapeutical applications. For this reason, FLUKA is being heavily benchmarked Sommerer et al. (2006) against models and experimental data concerning ion beams of interest for hadrontherapy. In Figure 2 an example of nice agreement between Bragg peak calculations and data is shown. The symbols represent LBL (circles) and GSI (triangles) experimental data Sihver et al. (1998), the line is the prediction of FLUKA. For the profile reproduction at large depths, nuclear interactions below $100 \mathrm{MeV} / \mathrm{n}$ play an important role. 

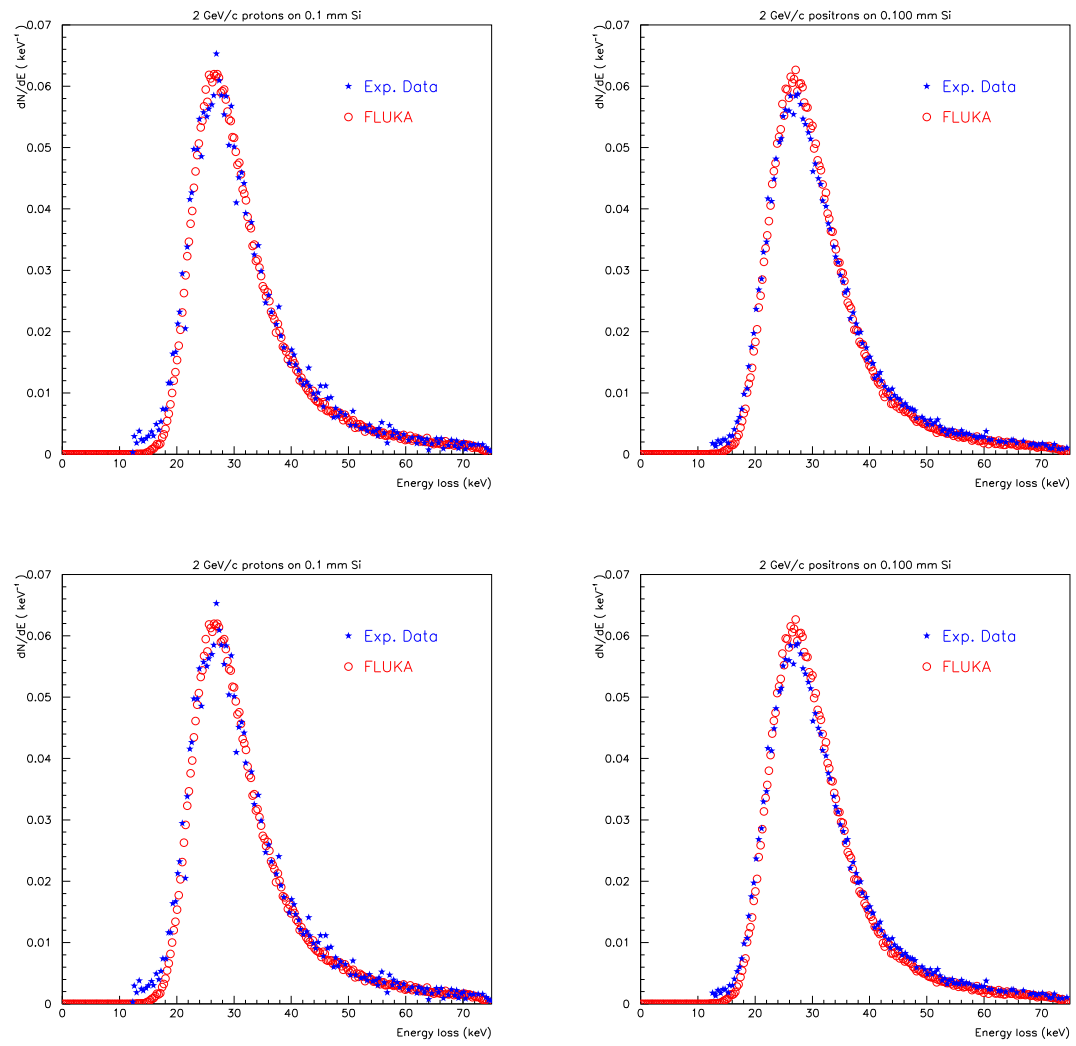

Figure 1: Energy deposition spectra for $2 \mathrm{GeV}$ protons (top) and positrons (bottom) on a $100 \mu \mathrm{m}$ thick Silicon detector.

\section{Hadronic interaction models}

A basic description of hadronic interactions in FLUKA and of their most recent developments can be found in Ferrari and Sala (1998); Collazuol et al. (2000); Battistoni et al. (2006a). Hadron-nucleon interactions at energies below a few $\mathrm{GeV}$ are simulated in FLUKA by the isobar model, through resonance production and decay, and by taking into account elastic, charge and strangeness exchange. Elementary hadron-hadron collisions at energies above a few $\mathrm{GeV}$ are described thanks to an implementation of the Dual Parton Model (DPM) Capella et al. (1994), coupled to a hadronization scheme. This model allows a successful description of soft collision processes that cannot be addressed by perturbative QCD.

Hadron-hadron collisions are the main building blocks of hadron-nucleus collisions. Multiple collisions of each hadron with the nuclear constituents are taken into account by means of the Glauber-Gribov calculus Glauber and Matthiae (1970); Gribov (1969). Particular efforts are devoted to the study of nuclear 


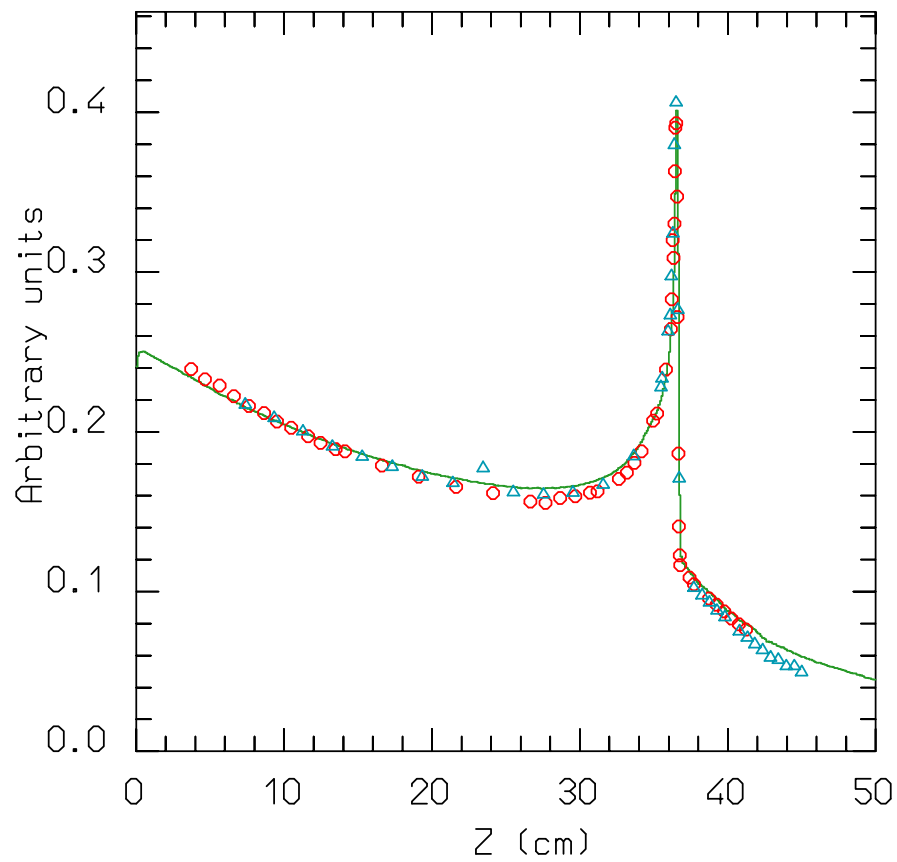

Figure 2: Dose versus depth distribution for $670 \mathrm{MeV} / \mathrm{n}{ }^{20} \mathrm{Ne}$ ions on a water phantom.

effects on hadron propagation. These are treated by the FLUKA nuclear interaction model called PEANUT Ferrari and Sala (1994); Fassò et al. (1995); Ferrari and Sala (1998); Battistoni et al. (2006a). This model includes a Generalized IntraNuclear Cascade (GINC) with smooth transition to a preequilibrium stage performed with standard assumptions on exciton number or excitation energy.

GINC modelling in PEANUT is highly sophisticated. Different nuclear densities are adopted for neutrons and protons, Fermi motion is defined locally including wave packet-like uncertainty smearing, the curvature of particle trajectories due to the nuclear potential is taken into account, binding energies are obtained from mass tables and updated after each particle emission, energymomentum conservation including the recoil of the residual nucleus is ensured. Quantum effects are explicitly included: Pauli blocking, formation zone, nucleon anti-symmetrization, nucleon-nucleon hard-core correlations, coherence length. Nuclear medium effects on the $\Delta$ resonance properties are accounted for when treating pion interactions Ferrari and Sala (1998); Fassò et al. (1995) and pion reinteractions in the nucleus. 

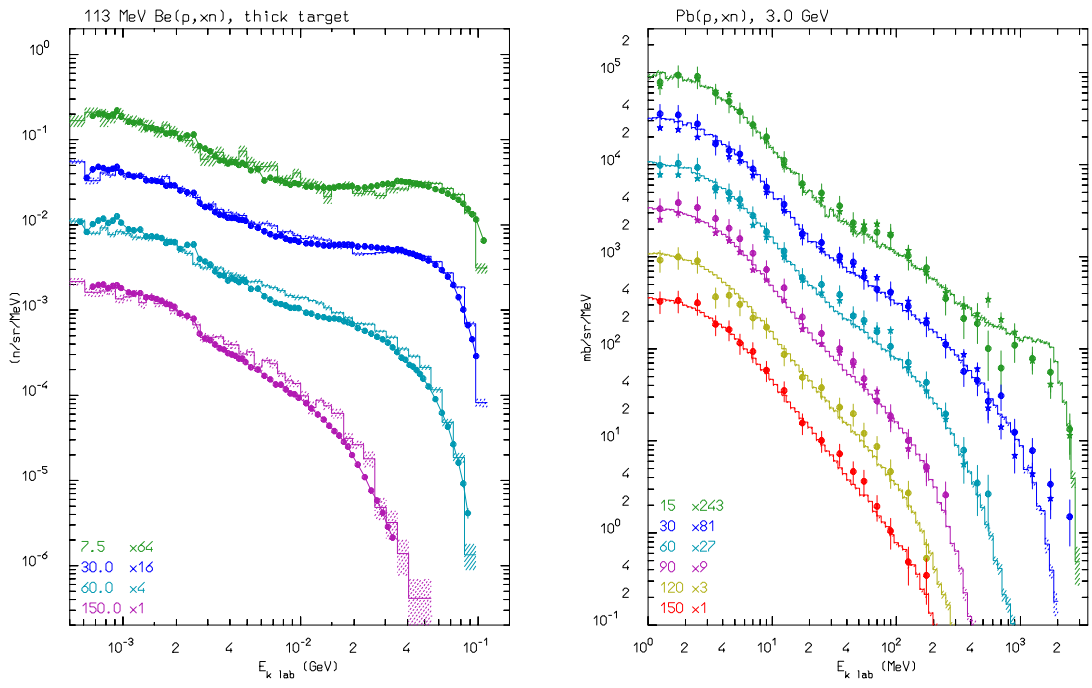

Figure 3: Emitted neutron spectra at different angles from interactions of $113 \mathrm{MeV}$ protons on a thick Beryllium target (top) and from $3 \mathrm{GeV}$ protons on a lead target (bottom).

The GINC step goes on until all nucleons are below a smooth threshold around $50 \mathrm{MeV}$, and all particles but nucleons (typically pions) have been emitted or absorbed. At the end of the GINC stage a few particles may have been emitted and the input configuration for the preequilibrium stage is characterized by the total number of protons and neutrons, by the number of particle-like excitons (nucleons excited above the Fermi level), and of hole-like excitons (holes created in the Fermi sea by the intranuclear cascade interactions), by the nucleus excitation energy and momentum. All the above quantities can be derived by proper counting of what occurred during the intranuclear cascade stage. The exciton formalism of FLUKA follows that of M. Blann and coworkers Blann (1971, 1972); Blann and Vonach (1983); Blann (1983), with some modifications:

- Inverse cross sections from systematics

- Correlation/formation zone/hardcore effects on reinteractions

- Constrained exciton state densities for the configurations $1 \mathrm{p}-1 \mathrm{~h}, 2 \mathrm{p}-1 \mathrm{~h}$, 1p-2h, 2p-2h, 3p-1h and 3p-2h

- Energy dependent form for the single particle density $g_{x}$ Shlomo (1992)

- Starting values for the position dependent parameters given by the point like ones as obtained out of the GINC stage.

- Angular distributions of emitted particles in the fast particle approximation 
For further details see ref. Ferrari and Sala (1998).

PEANUT has proved to be a precise and reliable tool for intermediate energy hadron-nucleus reactions. Its "nuclear environment" is also used in the modelization of (real and virtual) photonuclear reactions, neutrino interactions, nucleon decays, muon captures.

Examples of PEANUT results on neutron production from low energy proton interactions are shown in Figure 3 (experimental data from Refs. Meier et al. (1989); Ishibashi et al. (1997)). These benchmarks are of relevance for, for instance, calorimetry. Indeed, even in showers initiated by high energy projectiles, most of the interactions occur at medium-low energies, and the amount of visible energy depends critically on the energy balance and neutron balance in low energy reactions. Emission of energetic light fragments through the coalescence process is included all along the PEANUT reaction chain. This allows to reproduce the high energy tail of the light fragment spectra, as in Fig. 4. A major improvement has been the extension of PEANUT to cover the whole energy range, replacing the simplified intranuclear cascade model that was used for projectile energies larger than $5 \mathrm{GeV}$. Results obtained with the latest FLUKA version are shown in Figs. 5, 6 and 7
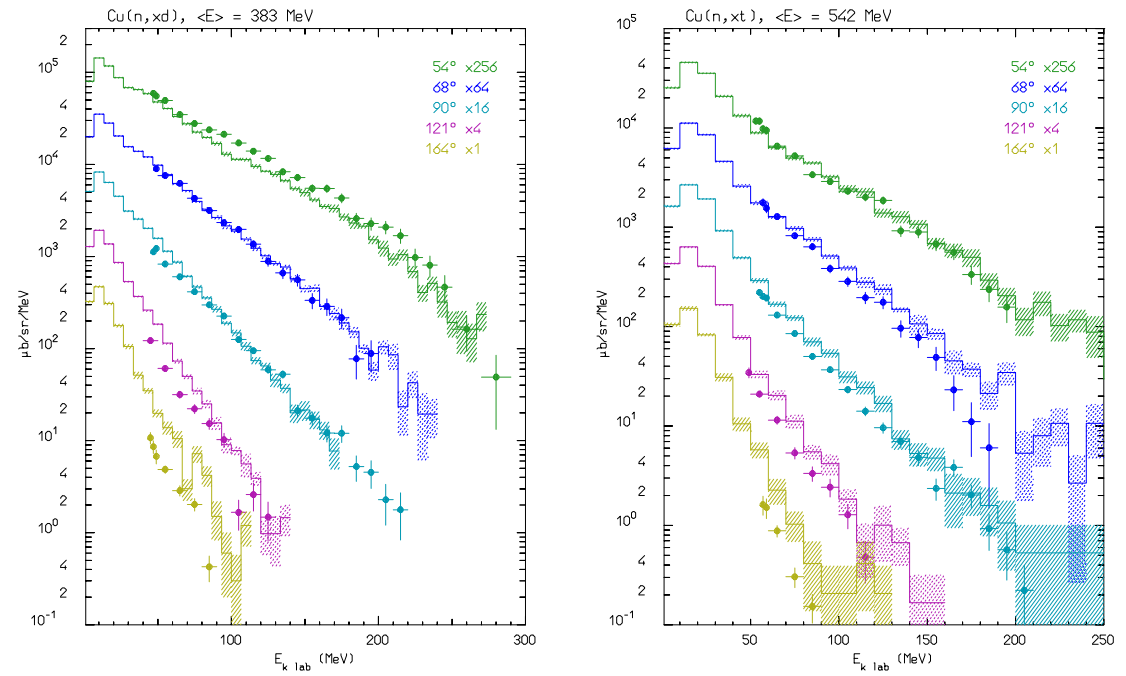

Figure 4: Deuteron (left) and triton (right) emission from $383 \mathrm{MeV}$ and $542 \mathrm{MeV}$ neutrons on $\mathrm{Cu}$ respectively (exp data from Franz et al. (1990))

The final steps of the reaction include evaporation in competition with fission and gamma deexcitation. For light nuclei, a Fermi break-up model is implemented. These equilibrium processes are critical for a correct calculation of residual nuclei distributions, as well as for the production of most neutrons. This topic is obviously important for activation and residual dose rate studies; it is also indirectly important for calorimetry: since the energy spent in breaking nuclear bonds is a major source of non-compensation and spread in energy deposition, a correct reproduction of residual nuclei distribution is a proof that 

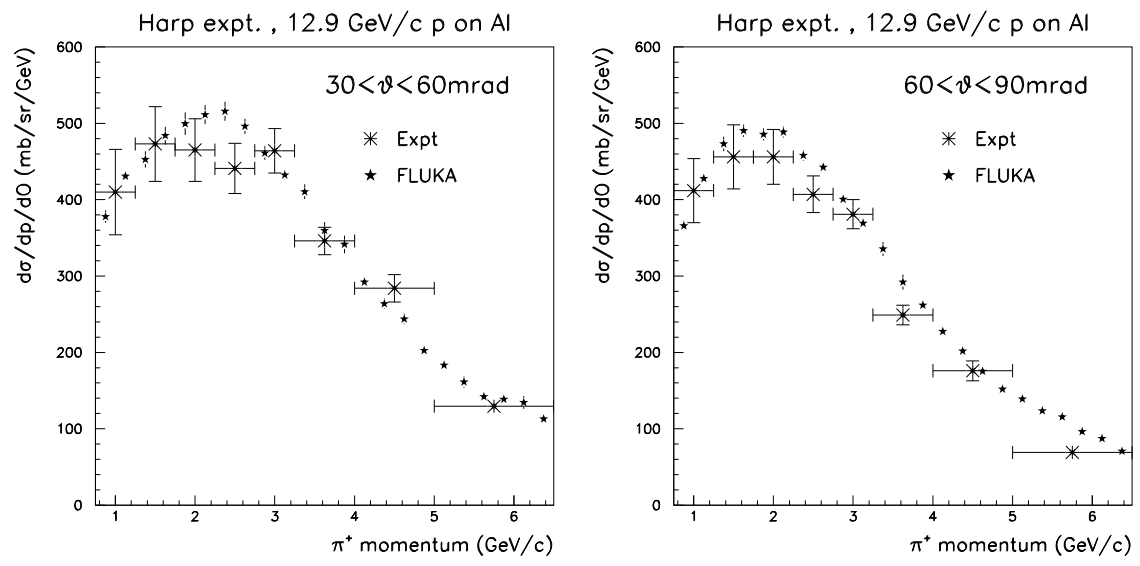

Figure 5: Computed $\pi^{+}$double differential production cross section for $12.9 \mathrm{GeV} / \mathrm{c}$ protons on Aluminum for different angular intervals, compared with experimental data Collaboration (2006).

binding energy losses are correctly taken into account. The FLUKA evaporation model, which is based on the Weisskopf-Ewing approach, has been continuously updated along the years, with the inclusion, for instance, of sub-barrier emission, full level density formula, analytic solution of the emission widths, evaporation of nuclear fragments up to $A \leq 24$.

Recent improvements in the treatment of fission and in the adopted level densities were particularly effective for the description of residual nuclei production from heavy targets. An example of the present code capabilities is shown in Fig. 8. More complex benchmarks have been carried out at the CERF Mitaroff and Silari (2002) facility at CERN. Samples of different materials have been irradiated with a mixed hadron field with broad energy spectrum. Comparison of activation and dose rate curves with FLUKA simulations Brugger et al. (2005) show very nice agreement, as for example in Fig. 9.

\section{Low energy neutrons}

Transport of neutrons with energies lower than $20 \mathrm{MeV}$ is performed in FLUKA by a multigroup algorithm. The multi-group technique, widely used in low-energy neutron transport programs, consists in dividing the energy range of interest in a given number of intervals ("energy groups") and computing with specialized codes the scattering matrices out of available neutron cross section evaluated data sets, taking into account all channels and proper cross section self-shielding. In the FLUKA cross-section library, the energy range is divided into 260 approximately equal logarithmic energy groups of approximately equal logarithmic width with 31 thermal groups. About 270 isotopes/materials are available to the user based on recent evaluated nuclear data files ENDF, JENDL, JEFF. Almost all materials are available at 2 temperatures $87 \mathrm{~K}$ and $296 \mathrm{~K}$. The 

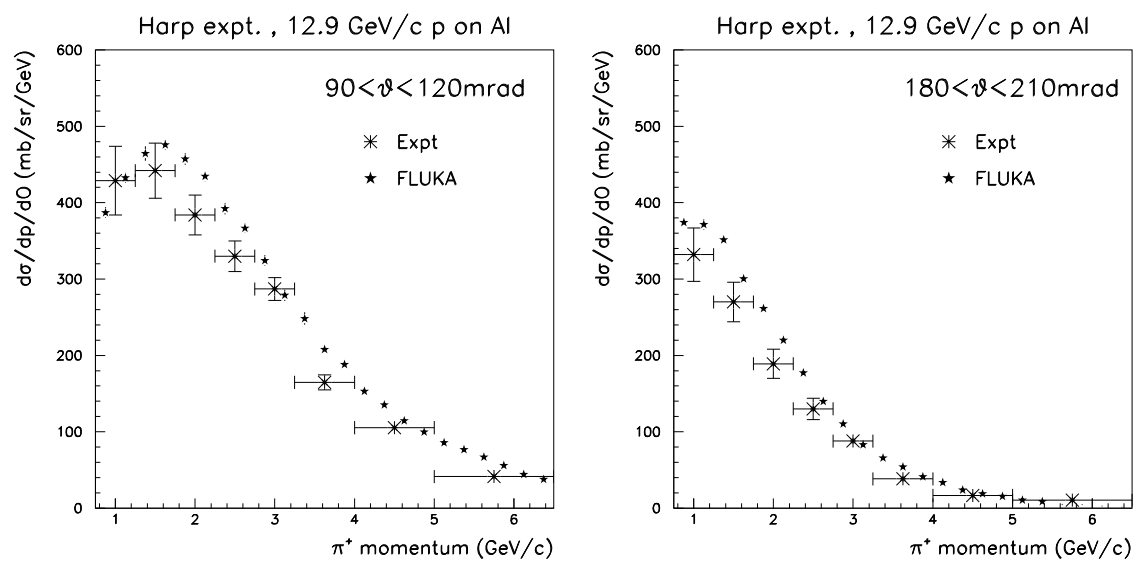

Figure 6: Same as Fig. 5 for other two angular intervals.

angular probabilities for inelastic scattering are obtained by a discretisation of a P5 Legendre polynomial expansion. For a few isotopes only, neutron transport can be done also using continuous (pointwise) cross-sections. For ${ }^{1} \mathrm{H},{ }^{6} \mathrm{Li},{ }^{10} \mathrm{~B}$, and ${ }^{40} \mathrm{Ar}$ it is applied as a user option (for all reactions in ${ }^{1} \mathrm{H},{ }^{6} \mathrm{Li}$, and ${ }^{40} \mathrm{Ar}$, and only for the reaction ${ }^{10} \mathrm{~B}(\mathrm{n}, \alpha){ }^{7} \mathrm{Li}$ in ${ }^{10} \mathrm{~B}$ ): in this case not only pointwise cross section are applied, but fully correlated particle production is accounted for, allowing to compute recoil spectra and possible correlated quantities (ie trigger/veto conditions). For the reaction ${ }^{14} \mathrm{~N}(\mathrm{n}, \mathrm{p}){ }^{14} \mathrm{C}$, pointwise neutron transport is always applied. In general, gamma generation by low-energy neutrons (but not gamma transport) is treated in the frame of a multigroup scheme too. A downscattering matrix provides the probability, for a neutron in a given energy group, to generate a photon in each of 42 gamma energy groups, covering the range $10 \mathrm{keV}$ to $20 \mathrm{MeV}$. In all cases, the generated gammas are transported in the same way as all other photons in FLUKA.

\section{Nucleus-Nucleus interactions}

Nucleus-nucleus interactions are performed through interfaces with external generators, also supported up to $10000 \mathrm{TeV} / \mathrm{n}$, thanks to the interfaces with a modified version of rQMD-2.4 Sorge et al. (1989); Andersen et al. (2004) for energies below $5 \mathrm{GeV} / \mathrm{n}$ and with DPMJET-III Roesler et al. (2001) at high energies. It is worth mentioning that the external generators, as well as the ones under development, share the same evaporation/deexcitation stage developed for hadron-nucleus interactions. Examples of results can be found in Andersen et al. (2004); Aiginger et al. (2005).

Work is in progress to complement and eventually substitute the rQMD interface with new QMD codes developed by the FLUKA collaboration Garzelli et al. (2006). The non-relativistic QMD model has already been interfaced to FLUKA and tests on thin and thick target data are in progress. 

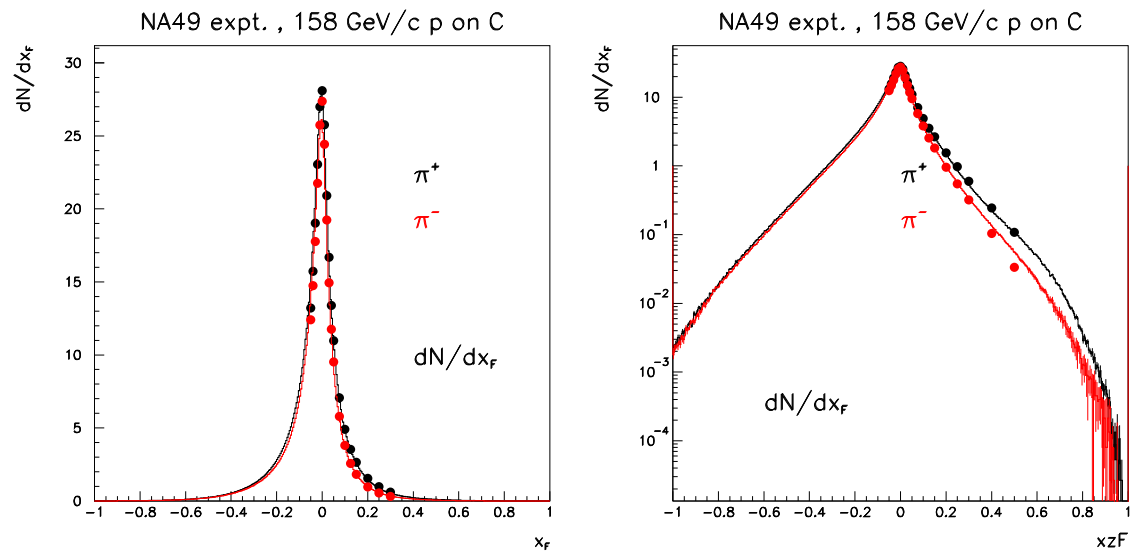

Figure 7: Feynman $-x$ distributions for $\pi^{+}$and $\pi^{-}$production for proton interactions on Carbon at $158 \mathrm{GeV} / \mathrm{c}$, as measured by NA49 Alt et al. (????) (symbols) and predicted by FLUKA (histograms). Linear scale on the left, logarithmic scale on the right.

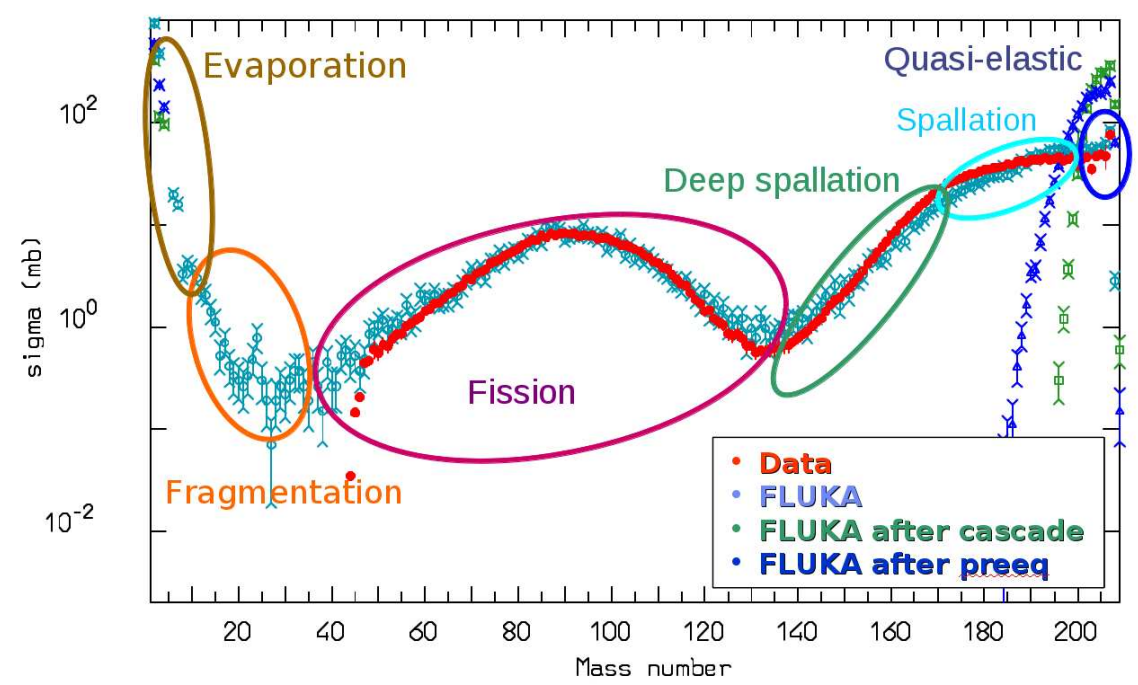

Figure 8: Residual nuclei production from $1 \mathrm{GeV}$ protons on Lead. Data from Enqvist et al. (2001)

For lower energies below $100 \mathrm{MeV} / \mathrm{n}$ the implementation of a Boltzmann Master Equation (BME) Cerutti et al. (2006) is used.

\section{Radiation Induced Damage}

The prediction of the structural damage to materials under irradiation is essential to evaluate consequences due to long term employment of construc- 

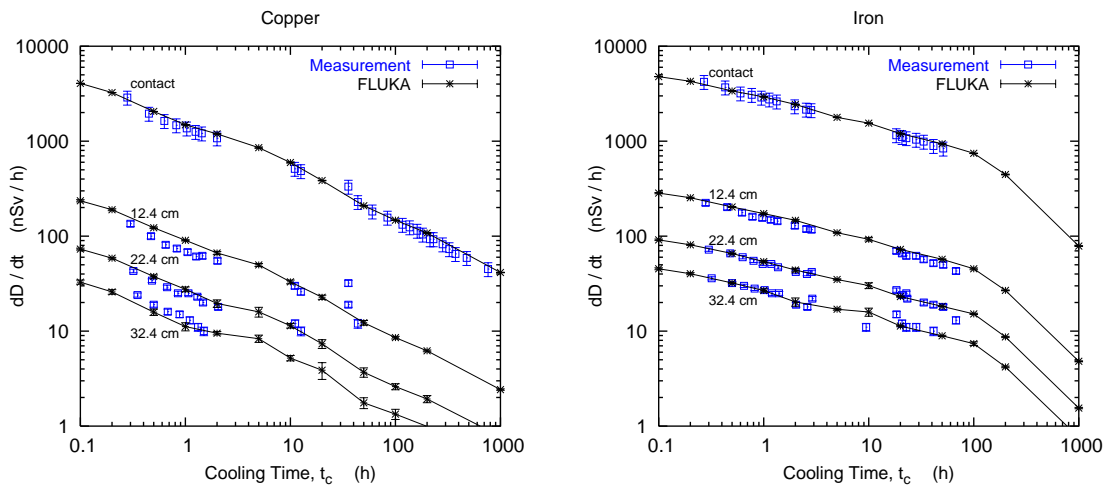

Figure 9: Dose rate as a function of cooling time for different distances between sample and detector. Left:Copper sample. Right: iron sample, both irradiated at the CERF facility at CERN Mitaroff and Silari (2002). Adapted from Brugger et al. (2005)

tion materials in nuclear reactors and charged particle accelerators. Radiation damage effects is implemented in FLUKA for all particles. In contrast with the implementation in other codes, we decided not to employ the Lindhard partition function but rather rework the formulas for an equivalent partition function restricted in energy above a user defined damage threshold, including relativistic corrections Fassò et al. (2011). The implementation is based on the integration of the universal potential using realistic form factors, allowing for calculation of the restricted nuclear energy losses for the recoils above a certain fraction of energy. The integration was performed in the reduced energy frame to remove any dependence on the projectile and target nucleus. Therefore, FLUKA is able to predict in an accurate way the number of recoils (and subsequent subcascade of the recoils) which has the power to induce damage to the material. Finally the conversion to dpa is performed by using a modified Kinchin-Pease damage model and taking into account the effect on the displacement efficiency for higher recoil energies due to recombination and migration of the Frenkel pairs. All charged particles including leptons, hadrons and heavy ions, benefit from the algorithm. However for neutron interactions, photo-nuclear interactions, Bremsstrahlung, pair production, Compton scattering the recoil nucleus is either calculated analytically or sampled from a distribution and is further subject to the above algorithm for the damage estimation.

\section{Geometry}

Transport in arbitrarily complex geometries, including magnetic field, can be accomplished using the FLUKA combinatorial geometry. This geometry can describe setups of arbitrary complexity and allows for repetition of objects, as well for run-time movement of some of them if required (ie for the modulating wheel of a therapy beam). A suitable voxel geometry module allows to model properly CT scans or other detailed 3D representations of human beings, typically for dosimetry or therapy planning purposes. 


\section{Flair: graphical user interface}

Flair V.Vlachoudis (2009) is a user-friendly graphical interface for FLUKA Monte Carlo transport code (Fig. 10). It provides an Integrated Development Environment (IDE) for all stages of FLUKA simulations, from building an error free input file, debugging, creation of user written routines, execution, status monitoring, data processing and plot generation. Flair is written in Python $2.5+$ van Rossum (????) (and is python 3.x compatible) using Tkinter as a graphical user interface. The use of the program greatly enhances the productivity of the users and provides much stepper learning curve for the beginners.

Flair is capable in importing and exporting in various file formats MCNP H.G.Hughes et al. (1999), GDML Chytracek (2001) as well technical drawings and super impose them on the FLUKA geometry for easier editing and cross checking.

The program and the source code can be freely downloaded from http://www.fluka.org/flair

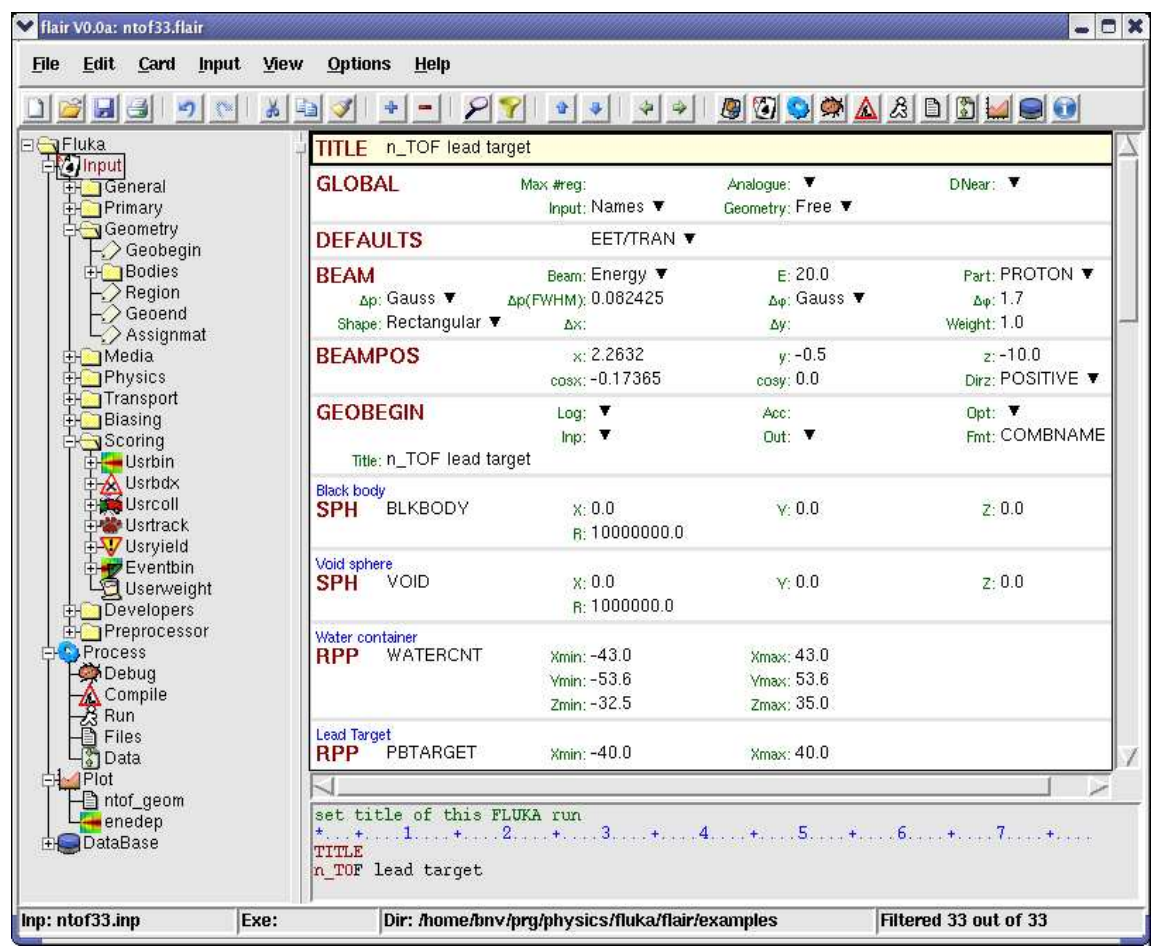

Figure 10: Flair graphical user interface

\subsection{Geometry editing}

Flair includes real-time geometry visualization and graphical editing, using a $\mathrm{C}++$ geometry engine as a python module (Fig. 11). With the dynamic layer mechanism which is implemented, the engine permits the user to create 
sophisticated views of various quantities overlaid on the geometry e.g. density maps, particle thresholds, superposition of technical drawings and images, scoring quantities from 3D grids (USRBIN), etc. The geometry engine operates in two modes: two dimensional cross section cuts and three dimensional projections. There is a full cross talk between the different modes of operation; the $3 \mathrm{D}$ view can be merged together with the $2 \mathrm{D}$ cross section plots. Special care has been taken to achieve the same numerical precision in all operations, using always the fastest method for each layer. Multiple geometry engines can run in parallel for the same geometry profiting from the modern multi-core CPU architectures.

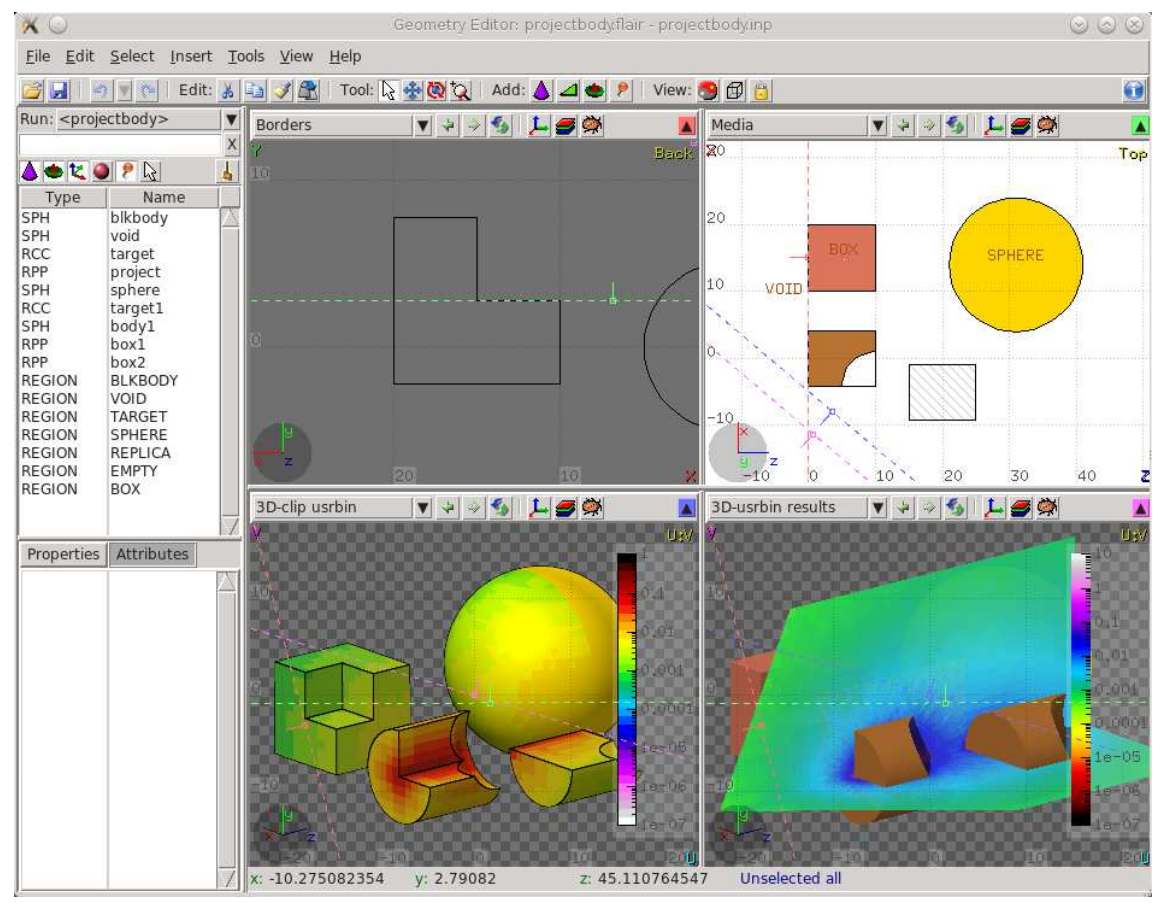

Figure 11: Flair geometry editor interface

\subsection{Medical applications}

The use of FLUKA is spreading also to medical applications, which provides a fundamental element in the early states of scanner designs. To facilitate the use of FLUKA for medical applications, flair integrates intuitive PET scanner geometry generator and importing routines for processing DICOM Association (1996) files. DICOM is an a non-proprietary data interchange protocol used in medicine. 3D CT scans, MRI, PET dose maps, as well dose distribution from the treatment planning systems all use the DICOM format to exchange their information. Flair is able to import the DICOM files (Fig. 12) and convert 
them in FLUKA voxel geometries, and/or 3D dose distributions to be used as a source for FLUKA.

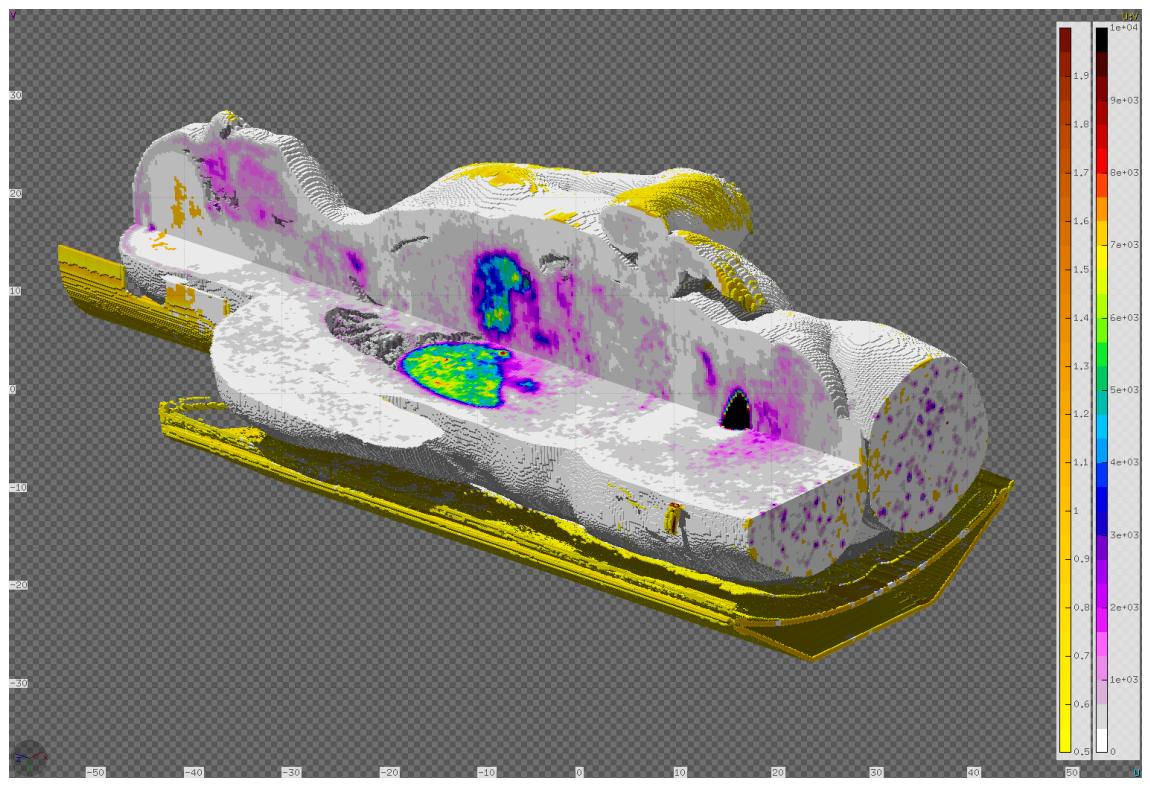

Figure 12: Flair importing of CT scans and PET dose distributions

\section{Applications}

The following sections show some illustrative examples demonstrating the capabilities of the FLUKA code.

\subsection{LHC collision debris}

The Large Hadron collider at CERN, during the first years of operation brought at continuous collision two $3.5 \mathrm{TeV}$ proton beams at four interaction points. Each beam stores a huge amount of energy of the order of $350 \mathrm{MJ}$, enough to melt half a ton of copper. The machine uses superconducting technology for the about 10000 magnets, where a tiny amount of energy deposited on their coils can bring them out of the superconducting state. Proton-proton interactions at $7 \mathrm{TeV}$ center of mass energy are performed by FLUKA through its interface to the DPMJET3 event generator. The particle showers impacting the BLMs are produced by primary collision products leaving the beam tube aperture, in particular energetic $(\mathrm{TeV})$ pions captured by the strong magnetic field in the quadrupoles. The collision debris of the two circulating beams can severely damage the superconducting final focusing triplet magnets if not a suitable protection system is used. FLUKA was extensively used for the optimization of such a protection system. Fig. 13 shows the signal the Beam Loss 
Monitors (BLM) will see from both the FLUKA predictions and from the measurements where a remarkable agreement is found despite the fact that we are concerned with the periphery of the cascade V.Boccone et al. (2013).

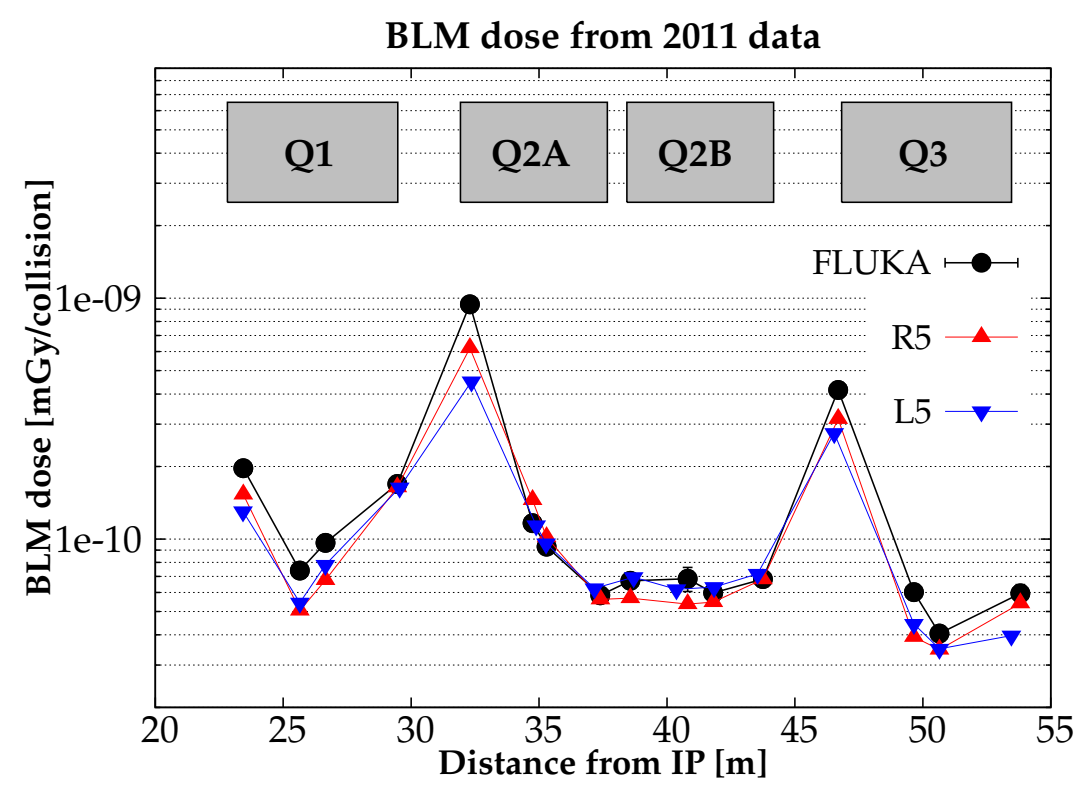

Figure 13: BLM measurements at IP5 during 3.5 TeV beam collisions and FLUKA predictions

\subsection{Prompt gamma imaging camera}

To reduce the uncertainty margins during the patient treatment with hadron beams different projects investigate the use of real-time prompt gamma cameras as a range control. To validate the concept a dedicated simulation setup was set using a PMMA target. The prompt gammas after traversing a collimator arrive to a slit camera based on NaI. The PMMA target has a cylindrical shape with $200 \mathrm{~mm}$ in height and $75 \mathrm{~mm}$ in radius, and is irradiated with a proton beam of $160 \mathrm{MeV}$. The lead collimator has $100 \mathrm{~mm}$ thickness and a gap of 50x50 $\mathrm{mm}^{2}$. Fig 14 shows the prompt photon energy spectrum after background subtraction as measured by the NaI detector Smeets et al. (2012) and the FLUKA predictions with a very nice agreement.

\section{Conclusions}

FLUKA is a multiparticle transport and interaction Monte Carlo code, able to work both in analog and biased mode. Its physical models are continuously upgraded and benchmarked against experimental data. It has a wide range of applications, in particle physics, but also in accelerator design and shielding, 


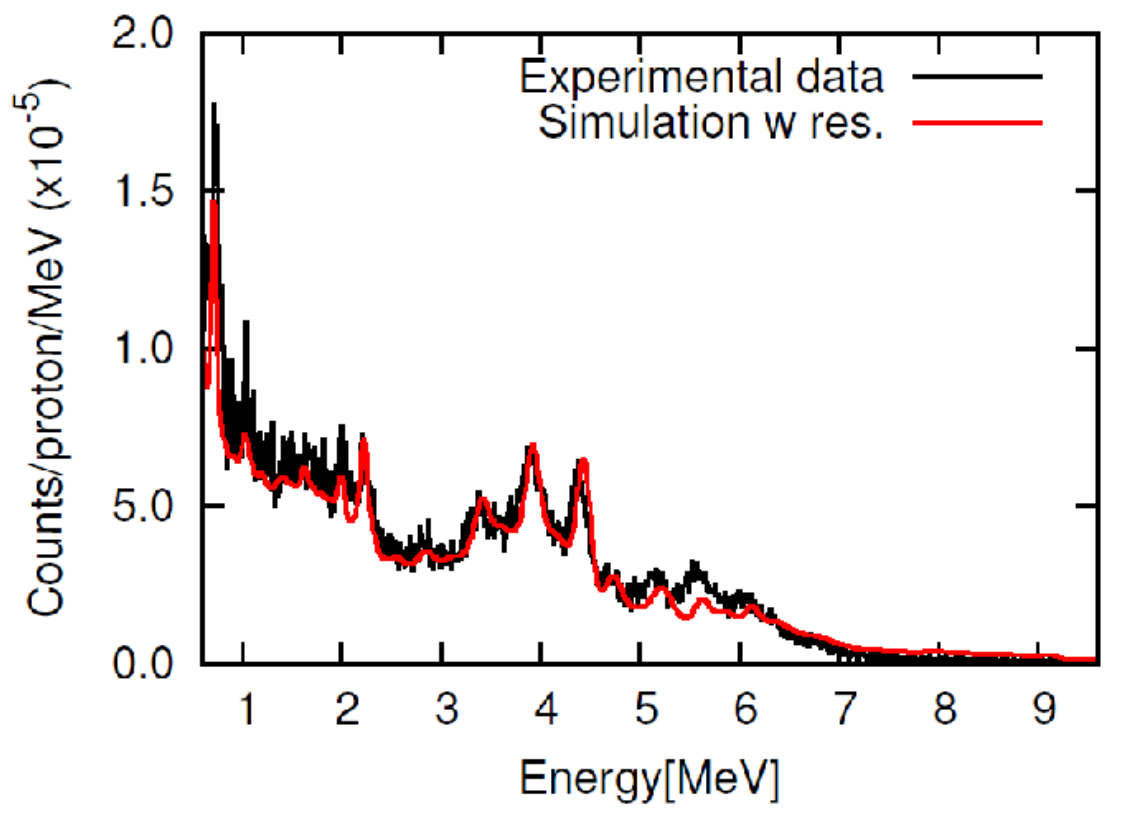

Figure 14: Energy spectrum of photons after background subtraction (collimator open vs collimator closed) for $160 \mathrm{MeV}$ p on PMMA. FLUKA red line, data black line (J.Smeets et al., IBA, ENVISION WP3)

dosimetry, radiation protection, hadrotherapy. In particular, it has proven capabilities in the simulation of particle detectors, ranging from big calorimeters, to neutron and radiation monitors.

\section{Acknowledgments}

This work has been carried out in the framework of the FLUKA Collaboration. This review paper is largely based on several presentations given by Collaboration members at International Conferences and Workshops. Part of this work was supported by Department of Energy contract DE-AC02-76SF00515.

Aiginger, H., et al., 2005. The fluka code: new developments and application to $1 \mathrm{gev} / \mathrm{n}$ iron beams. In: Adv. Space Res. Vol. 35. pp. 214-222.

Alt, C., et al., ???? Inclusive production of charged pions in $\mathrm{p}+\mathrm{c}$ collisions at 158-gev/c beam momentum. arXiv:hep-ex/0606028.

Andersen, V., et al., 2004. The fluka code for space applications: recent developments. In: Adv. Space Res. Vol. 34. p. 1302.

Association, N. E. M., 1996. Digital imaging and communications in medicine (dicom). NEMA Standard Publications PS 3. 
Bak, J., et al., 1987. Nucl. Phys. B 288, 681.

Battistoni, G., Cerutti, F., Ferrari, A., 2006a. The physics of high energy reactions. In: Gadioli, E. (Ed.), 11th Int. Conf. on Nuclear Reaction Mechanisms. p. 483.

Battistoni, G., Muraro, S., Sala, P., Cerutti, F., Ferrari, A., Roesler, S., Fassò, A., Ranft, J., September 6-8 2006b. The fluka code: Description and benchmarking. In: Albrow, M., Raja, R. (Eds.), Hadronic Shower Simulation Workshop 2006. Vol. 896 of AIP Conference Proceedings. Fermilab, U.S.A., pp. $31-49$.

Blann, M., 1971. Hybrid model for pre-equilibrium decay in nuclear reactions. Phys. Rev. Lett. 27, 337.

Blann, M., 1972. Importance of the nuclear density distribution on preequilibrium decay. Phys. Rev. Lett. 28, 757.

Blann, M., 1983. Precompound analyses of spectra and yields following nuclear capture of stopped $\pi^{-}$. Phys. Rev. C 28, 1648.

Blann, M., Vonach, H., 1983. Global test of modified precompound decay models. Phys. Rev. C 28, 1475.

Brugger, M., et al., 2005. In: Radiat. Prot. Dosim. Vol. 116. pp. 12-15.

Capella, A., Sukhatme, U., Tan, C.-I., Van, J. T. T., 1994. Dual parton model. Phys. Rep. 236, 225.

Cerutti, F., et al., 2006. In: Journ. of Phys.: Conference Series. Vol. 41. p. 212.

Chytracek, R., 2001. The geometry description markup language. Proc. Computing in High Energy and Nuclear Physics.

Collaboration, T. H., 2006. Nucl. Phys. B732, 1.

Collazuol, G., Ferrari, A., Guglielmi, A., Sala, P., 2000. Hadronic models and experimental data for the neutrino beam production. Nucl. Instr. Meth. A 449,609 .

Cullen, D., Hubbell, J., Kissel, L., 1997. Epdl97: the evaluated photon data library, '97 version. Tech. Rep. UCRL-50400, Vol. 6, Rev. 5.

Enqvist, T., et al., 2001. Isotopic yields and kinetic energies of primary residues in 1 a gev 208pb. Nucl. Phys. A686, 481.

Fassò, A., Ferrari, A., Ranft, J., Sala, P., 1995. Fluka: Performances and applications in the intermediate energy range. In: Specialists' Meeting on Shielding Aspects of Accelerators, Targets \& Irradiation Facilities. pp. 287-304. 
Fassò, A., Ferrari, A., Sala, P., 1997. New developments in fluka modelling of hadronic and em interactions. In: Hirayama, H. (Ed.), SARE-3. KEK report Proceedings $97-5$, p. 32.

Fassò, A., Ferrari, A., Sala, P., October 23-26 2001. Electron-photon transport in fluka: status. In: Kling, A., Barao, F., Nakagawa, M., T'avora, L. (Eds.), Monte Carlo 2000 Conference. Springer-Verlag Berlin, Lisbon, Portugal, pp. 159-164.

Fassò, A., Ferrari, A., Smirnov, G., Sommerer, F., Vlachoudis, V., 2011. Fluka realistic modeling of radiation induced damage. Progress in NUCLEAR SCIENCE and TECHNOLOGY 2, 769-775.

Ferrari, A., Sala, P., 1994. A new model for hadronic interactions at intermediate energies for the fluka code. In: MC93 Int. Conf. on Monte-Carlo Simulation in High-Energy and Nuclear Physics. World Scientific, p. 277.

Ferrari, A., Sala, P., 1998. The physics of high energy reactions. In: Gandini, A., Reffo, G. (Eds.), Workshop on Nuclear Reaction Data and Nuclear Reactors Physics, Design and Safety. p. 424.

Ferrari, A., Sala, P., Fassò, A., Ranft, J., 2005. Fluka: a multi-particle transport code. Tech. Rep. CERN-2005-10, INFN/TC-05/11, SLAC-R-773.

Ferrari, A., Sala, P., Guaraldi, G., Padoani, F., 1992. An improved multiple scattering model for charged particle transport. Nucl. Instr. Meth. B 71, 412426.

Franz, J., P. Koncz, E. R., et al., 1990. Neutron-induced production of protons, deuterons and tritons on copper and bismuth. Nucl. Phys. A510, 774.

Garzelli, M., et al., 2006. In: Physics Conference Series. Vol. 41. p. 519.

Glauber, R., Matthiae, G., 1970. High-energy scattering of protons by nuclei. Nucl. Phys. B 21, 135.

Gribov, V., 1969. Glauber corrections and the interaction between high-energy hadrons and nuclei. Sov. Phys. JETP 29, 483.

H.G.Hughes, et al., Sept 27-30 1999. Mcnpx for neutron-proton transport. In: Math. \& Comp. ANS. Madrid Spain.

Ishibashi, K., et al., 1997. J. Nucl. Sci. Technol. 34, 529.

Meier, M., Clark, D., Goulding, C., McClelland, J., Morgan, G., Moss, C., 1989. Nucl. Sci. and Eng. 102, 310.

Mitaroff, A., Silari, M., 2002. Rad. Prot. Dosim. 102, 7-22.

Roesler, S., Engel, R., Ranf, J., 2001. The monte carlo event generator dpmjetiii. In: Proc. Monte-Carlo 2000 Conference. Springer-Verlag Berlin, p. 1033. 
Shlomo, S., 1992. Energy level density of nuclei. Nucl. Phys. A 539, 17.

Sihver, L., et al., 1998. Jpn. J. Med. Phys. 18, 1.

Smeets, J., et al., 2012. Prompt gamma imaging with a slit camera for real-time range control in proton therapy. Phys. Med. Biol. 57, 3371.

Sommerer, F., Parodi, K., Ferrari, A., Poljanc, K., Enghardt, W., Aiginger, H., 2006. Phys. Med. Biol. 51, 4385.

Sorge, H., H.Stocker, Greiner, W., 1989. Relativistic quantum molecular dynamics approach to nuclear collisions at ultrarelativistic energies. Nucl. Phys. A498, 567c.

van Rossum, G., ???? Python programming language http://www.python.org. URL http://www . python.org

V.Boccone, et al., 2013. Beam-machine interaction at the cern lhc. In: Nuclear Data.

V.Vlachoudis, 2009. Flair: A powerful but user friendly graphical interface for fluka. In: Int. Conf. on Math, Comp Meth \& Reactor Physics. Saratoga Springs, NY. 